\title{
Correction to: The use of multivariate statistical methods for optimization of the surface water quality network monitoring in the Paraopeba river basin, Brazil
}

\author{
Giovanna Moura Calazans • Carolina Cristiane Pinto • \\ Elizângela Pinheiro da Costa • Anna Flávia Perini • \\ Sílvia Corrêa Oliveira $\mathbb{D}$
}

Published online: 24 August 2018

(C) Springer Nature Switzerland AG 2018

Correction to: Environ Monit Assess (July 2018) 190:491

https://doi.org/10.1007/s10661-018-6873-2

Unfortunately, the original version of this article was published online with error. The Tables 3 and 4 data was mixed up.

The corrected Tables 3 and 4 are shown in the next page. The original article has been corrected.

The online version of the original article can be found at https://doi.org/10.1007/s10661-018-6873-2

G. M. Calazans - C. C. Pinto - E. P. da Costa - A. F. Perini • S. C. Oliveira $(\square)$

Escola de Engenharia, Universidade Federal de Minas Gerais, Campus Pampulha, Av. Antônio Carlos, 6627, Bloco 1, sala 4525, Belo Horizonte, MG 31270-901, Brazil

e-mail: silvia@desa.ufmg.br 
Table 3 Surface water quality standards set in Normative Deliberation COPAM/CERH-MG 01/2008

\begin{tabular}{|c|c|c|c|c|}
\hline \multirow[t]{2}{*}{ Parameter } & \multirow[t]{2}{*}{ Unit } & \multicolumn{3}{|l|}{ Class } \\
\hline & & 1 & 2 & 3 \\
\hline Biochemical oxygen demand & mg. $\mathrm{L}^{-1} \mathrm{O}_{2}$ & 3 & 5 & 10 \\
\hline Chlorophyll-a & $\mu \mathrm{g} . \mathrm{L}^{-1}$ & 10 & 30 & 60 \\
\hline Density of cyanobacteria & cel.mL $\mathrm{mL}^{-1}$ & 20000 & 50000 & 100000 \\
\hline Dissolved copper & mg. $\mathrm{L}^{-1} \mathrm{Cu}$ & 0.009 & 0.009 & 0.013 \\
\hline Dissolved iron & mg. $\mathrm{L}^{-1} \mathrm{Fe}$ & 0.3 & 0.3 & 5.0 \\
\hline Dissolved oxygen & mg. $\mathrm{L}^{-1} \mathrm{O}_{2}$ & 6 & 5 & 4 \\
\hline Free cyanide & mg. $\mathrm{L}^{-1} \mathrm{CN}$ & 0.005 & 0.005 & 0.022 \\
\hline Nitrate & mg. $\mathrm{L}^{-1} \mathrm{~N}$ & 10.0 & 10.0 & 10.0 \\
\hline Nitrite & mg. $\mathrm{L}^{-1} \mathrm{~N}$ & 1 & 1 & 1 \\
\hline Oils and greases & & 0 & 0 & 0 \\
\hline $\mathrm{pH}$ & & 6 to 9 & 6 to 9 & 6 to 9 \\
\hline Total Ammoniacal Nitrogen $\mathrm{pH} \leq 7.5$ & mg. $\mathrm{L}^{-1} \mathrm{~N}$ & 3.7 & 3.7 & 13.3 \\
\hline T. Ammoniacal Nitrogen $7.5<\mathrm{pH}<8.0$ & mg. $\mathrm{L}^{-1} \mathrm{~N}$ & 2 & 2 & 5.6 \\
\hline T. Ammoniacal Nitrogen $8.0<\mathrm{pH}<8.5$ & mg. $\mathrm{L}^{-1} \mathrm{~N}$ & 1 & 1 & 2.2 \\
\hline T. Ammoniacal Nitrogen $\mathrm{pH} \geq 8.5$ & mg. $\mathrm{L}^{-1} \mathrm{~N}$ & 0.5 & 0.5 & 1 \\
\hline Thermotolerant coliforms & org. $100 \mathrm{~mL}^{-1}$ & 200 & 1000 & 4000 \\
\hline Total arsenic & mg. $L^{-1}$ As & 0.01 & 0.01 & 0.033 \\
\hline Total barium & mg. $\mathrm{L}^{-1} \mathrm{Ba}$ & 0.7 & 0.7 & 1.0 \\
\hline Total Boron & mg. $\mathrm{L}^{-1} \mathrm{~B}$ & 0.5 & 0.5 & 0.75 \\
\hline Total Cadmium & $\mathrm{mg} \cdot \mathrm{L}^{-1} \mathrm{Cd}$ & 0.001 & 0.001 & 0.01 \\
\hline Total Chloride & mg. $\mathrm{L}^{-1} \mathrm{Cl}$ & 250 & 250 & 250 \\
\hline Total chromium & mg. $\mathrm{L}^{-1} \mathrm{Cr}$ & 0.05 & 0.05 & 0.05 \\
\hline Total Dissolved Solids & mg. $\mathrm{L}^{-1}$ & 500 & 500 & 500 \\
\hline Total lead & mg. $\mathrm{L}^{-1} \mathrm{~Pb}$ & 0.01 & 0.01 & 0.033 \\
\hline Total manganese & $\mathrm{mg} \cdot \mathrm{L}^{-1} \mathrm{Mn}$ & 0.1 & 0.1 & 0.5 \\
\hline Total Nickel & mg. $\mathrm{L}^{-1} \mathrm{Ni}$ & 0.025 & 0.025 & 0.025 \\
\hline Total phosphorus & $\mathrm{mg} \cdot \mathrm{L}^{-1} \mathrm{P}$ & 0.1 & 0.1 & 0.15 \\
\hline Total selenium & mg. $\mathrm{L}^{-1} \mathrm{Se}$ & 0.01 & 0.01 & 0.05 \\
\hline Total Suspended Solids & mg. $\mathrm{L}^{-1}$ & 50 & 100 & 100 \\
\hline Total Zinc & mg. $\mathrm{L}^{-1} \mathrm{Zn}$ & 0.18 & 0.18 & 5 \\
\hline True color & mgPt.L ${ }^{-1}$ & - & 75 & 75 \\
\hline Turbidity & NTU & 40 & 100 & 100 \\
\hline
\end{tabular}


Table 4 Number of critical parameters for each of the monitoring sites in the Paraopeba river basin

\begin{tabular}{|c|c|c|c|c|c|c|c|c|c|c|}
\hline & \multicolumn{10}{|c|}{ Number of critical parameters } \\
\hline & $\underline{8}$ & $\underline{5}$ & $\underline{4}$ & $\underline{3}$ & 2 & & & 1 & & \\
\hline \multirow[t]{4}{*}{ Monitoring sites } & $\underline{\mathrm{BP} 073}$ & $\underline{\mathrm{BP} 069}$ & $\underline{\mathrm{BP} 098}$ & $\underline{\mathrm{BP} 071}$ & ВР024 & BP066 & $\underline{\mathrm{BP} 074}$ & ВР022 & $\underline{\mathrm{BP} 082}$ & BP096 \\
\hline & & BP084 & & BP080 & ВР027 & BP068 & BP076 & ВР026 & BP088 & BP099 \\
\hline & & & & $\underline{\mathrm{BP} 086}$ & ВР029 & $\underline{\mathrm{BP} 070}$ & ВР079 & ВP032 & $\underline{\mathrm{BP} 090}$ & \\
\hline & & & & & ВР036 & $\mathrm{BP} 072$ & ВР083 & ВР078 & BP092 & \\
\hline
\end{tabular}

Italic and underlined: monitoring sites belong to groups 1 to 6 (cluster analysis)

Upright and underlined: monitoring sites belong to group 7

Upright and no underline: monitoring sites belong to group 8 